\title{
Attaining Low Lattice Thermal Conductivity in Half-Heusler Sublattice Solid Solutions: Which Substitution Site Is Most Effective?
}

\author{
Rasmus Tranås ${ }^{1}\left(\mathbb{D}\right.$, Ole Martin Løvvik ${ }^{2,3}$ (D) and Kristian Berland ${ }^{1, *(D)}$ \\ 1 Department of Mechanical Engineering and Technology Management, Norwegian University of Life Sciences, \\ NO-1432 Ås, Norway; rasmus.andre.tranas@nmbu.no \\ 2 Centre for Materials Science and Nanotechnology, Department of Physics, University of Oslo, \\ NO-0316 Oslo, Norway; olemartin.lovvik@sintef.no \\ 3 SINTEF Sustainable Energy Technology, NO-0314 Oslo, Norway \\ * Correspondence: kristian.berland@nmbu.no; Tel.: +47-456-792-996
}

check for updates

Citation: Tranås, R.; Løvvik, O.M.; Berland, K. Attaining Low Lattice

Thermal Conductivity in

Half-Heusler Sublattice Solid

Solutions: Which Substitution Site Is Most Effective?. Electron. Mater. 2022,

3,1-14. https://doi.org/10.3390/

electronicmat3010001

Academic Editor: Ichiro Terasaki

Received: 15 November 2021

Accepted: 16 December 2021

Published: 5 January 2022

Publisher's Note: MDPI stays neutral with regard to jurisdictional claims in published maps and institutional affiliations.

Copyright: (c) 2022 by the authors. Licensee MDPI, Basel, Switzerland. This article is an open access article distributed under the terms and conditions of the Creative Commons Attribution (CC BY) license (https:/ / creativecommons.org/licenses/by/ $4.0 /)$.

\begin{abstract}
Low thermal conductivity is an important materials property for thermoelectricity. The lattice thermal conductivity (LTC) can be reduced by introducing sublattice disorder through partial isovalent substitution. Yet, large-scale screening of materials has seldom taken this opportunity into account. The present study aims to investigate the effect of partial sublattice substitution on the LTC. The study relies on the temperature-dependent effective potential method based on forces obtained from density functional theory. Solid solutions are simulated within a virtual crystal approximation, and the effect of grain-boundary scattering is also included. This is done to systematically probe the effect of sublattice substitution on the LTC of 122 half-Heusler compounds. It is found that substitution on the three different crystallographic sites leads to a reduction of the LTC that varies significantly both between the sites and between the different compounds. Nevertheless, some common criteria are identified as most efficient for reduction of the LTC: The mass contrast should be large within the parent compound, and substitution should be performed on the heaviest atoms. It is also found that the combined effect of sublattice substitution and grain-boundary scattering can lead to a drastic reduction of the LTC. The lowest LTC of the current set of half-Heusler compounds is around $2 \mathrm{~W} / \mathrm{Km}$ at $300 \mathrm{~K}$ for two of the parent compounds. Four additional compounds can reach similarly low LTC with the combined effect of sublattice disorder and grain boundaries. Two of these four compounds have an intrinsic LTC above $\sim 15 \mathrm{~W} / \mathrm{Km}$, underlining that materials with high intrinsic LTC could still be viable for thermoelectric applications.
\end{abstract}

Keywords: half-Heusler; lattice thermal conductivity; alloying; optimal substitution site; density functional theory; temperature-dependent effective potential

\section{Introduction}

The ability to convert excess heat into electricity and vice versa makes thermoelectricity interesting for a range of niche applications requiring local cooling or electricity generation. More effective thermoelectric materials could increase the applicability of thermoelectricity and thereby contribute to reducing energy consumption and carbon emissions [1,2]. The figure-of-merit of a thermoelectric material is at an operational temperature $T$ given by

$$
Z T=\frac{\sigma S^{2} T}{\kappa_{e}+\kappa_{\ell}},
$$

where $S$ is the Seebeck coefficient, $\sigma$ is the electrical conductivity, and $\kappa_{e}$ and $\kappa_{\ell}$ are the electronic and lattice thermal conductivity (LTC), respectively. The electronic contributions to ZT exhibit conflicting dependency of the charge carrier concentration, requiring opti- 
mization of the doping level [3-6], though there has been significant progress in recent years [7-10].

Alloying, i.e., formation of solid solutions, is a widely used approach to modify materials, including their thermoelectric, optoelectronic [11,12], and electrolytic properties [13,14]. It can also be used to modify band gaps, defect formation densities, and structural phase transitions [15-19]. Introducing additional elements can, however, complicate materials synthesis e.g., by undesirable formation of secondary phases [20-26]. Still, much progress has been made in ensuring single-phase alloys for certain material classes [27] including half-Heuslers [28-30].

Half-Heusler $(\mathrm{HH})$ compounds are ternary compounds with an XYZ composition, where the $X Z$ sublattice forms a rocksalt structure and the $Y Z$ sublattice represents a zinc-blende structure [31]. The thermoelectric properties of HHs have been intensively studied [32-44]. While their electronic properties tend to be beneficial, they typically suffer from high LTC [45].

For optimizing thermoelectric properties, many studies have focused on identifying alloys with band structures that can provide a high power factor $\mathcal{P}=\sigma S^{2}$ without increasing $\kappa_{e}$ too much [46-48]. For such materials, the problem is reduced to that of lowering $\kappa_{\ell}$ while maintaining beneficial electronic properties [6]. An efficient strategy to reduce the LTC is to introduce sublattice disorder, for instance through partial isovalent atomic substitutions on one or more of the sublattices of the compound [49-54]. Such substitutions can be effective as they often have a limited effect on the electronic mobility [55-58] and hence the attainable $\mathcal{P}$.

Several studies have explored partial substitution on the X-site of HHs, e.g., replacing a fraction of $\mathrm{Zr}$ in $\mathrm{ZrNiSn}$ by Ti or Hf [59-62], or replacing a fraction of $\mathrm{Nb}$ in $\mathrm{NbCoSn}$ by $\mathrm{Sc}$ or $\mathrm{Ti}[63,64]$. Z-site substitution has also been investigated in $\mathrm{ZrCoSb}$, replacing Sb by Sn $[65,66]$. It would be useful to establish a systematic overview of the effectiveness of substitutions at different sites at reducing the LTC. An indicator of this can be provided by the magnitude of the mass-variance parameter [67], given as

$$
g_{i}=\sum_{j} c_{i}^{j}\left(\frac{m_{i}^{j}-\bar{m}_{i}}{\bar{m}_{i}}\right)^{2} .
$$

Here, $c_{i}^{j}$ is the concentration of atom $j$ substituting atom $i, m_{i}^{j}$ is the mass of the substituent atom, and $\bar{m}_{i}=\sum_{j} c_{i}^{j} m_{i}^{j}$ is the average atomic mass. However, the nature of the phonon modes also plays a significant role, as exemplified for $\mathrm{Ti}_{x} \mathrm{Zr}_{y} \mathrm{Hf}_{1-x-y} \mathrm{NiSn}$ in Ref. [61]. In this paper, we use first-principles calculations to assess the change in LTC when introducing sublattice disorder on each of the three crystallographic sites of $122 \mathrm{HHs}$

\section{Methods}

\subsection{Thermal Transport and Phonon Scattering Mechanisms}

The LTC is calculated with the temperature-dependent effective potential (TDEP) approach $[68,69]$, in which the finite-temperature second- and third-order force constants are extracted based on atomic displacements and forces. Within phonon Boltzmann transport theory employing the relaxation-time approximation, the LTC is given by

$$
\kappa_{\ell}=\frac{1}{V} \sum_{\lambda} c_{\lambda} v_{\lambda}^{2} \tau_{\lambda}^{\mathrm{tot}},
$$

where $\lambda=(\mathbf{q} s)$ indicates the wave vector $\mathbf{q}$ and the phonon mode $s$. Further, $c_{\lambda}$ is the phonon heat capacity, $v_{\lambda}$ the phonon group velocity, and $\tau_{\lambda}^{\text {tot }}$ the total phonon relaxation time. 
The scattering rate from three-phonon scattering is given by

$$
\begin{aligned}
\frac{1}{\tau_{\lambda}}= & \frac{\hbar \pi}{8} \sum_{\lambda^{\prime} \lambda^{\prime \prime}}\left|\Phi_{\lambda \lambda^{\prime} \lambda^{\prime \prime}}\right|^{2}\left[\left(n_{\lambda^{\prime}}+n_{\lambda^{\prime \prime}}+1\right) \delta\left(\omega_{\lambda}-\omega_{\lambda^{\prime}}-\omega_{\lambda^{\prime \prime}}\right)\right. \\
& \left.+2\left(n_{\lambda^{\prime}}-n_{\lambda^{\prime \prime}}\right) \delta\left(\omega_{\lambda}-\omega_{\lambda^{\prime}}+\omega_{\lambda^{\prime \prime}}\right)\right] .
\end{aligned}
$$

Here, $n$ is the equilibrium phonon distribution function, and $\Phi_{\lambda \lambda^{\prime} \lambda^{\prime \prime}}$ are the threephonon matrix elements,

$$
\Phi_{\lambda \lambda^{\prime} \lambda^{\prime \prime}}=\sum_{i j k} \sum_{\alpha \beta \gamma} \frac{\epsilon_{\alpha i}^{\lambda} \epsilon_{\beta j}^{\lambda^{\prime}} \epsilon_{\gamma k}^{\lambda^{\prime \prime}}}{\sqrt{m_{i} m_{j} m_{j}} \sqrt{\omega_{\lambda} \omega_{\lambda^{\prime}} \omega_{\lambda^{\prime \prime}}}} \times \Phi_{i j k}^{\alpha \beta \gamma} e^{i\left(\mathbf{q r}_{i}+\mathbf{q}^{\prime} \mathbf{r}_{j}+\mathbf{q}^{\prime \prime} \mathbf{r}_{k}\right)}
$$

Here, $i j k$ are indices of atoms, $\alpha \beta \gamma$ are Cartesian coordinates, $m_{i}$ is the mass of atom $i$, $\epsilon_{\alpha i}^{\lambda}$ is component $\alpha$ of the eigenvector for mode $\lambda$ and atom $i, \mathbf{r}_{i}$ is the lattice vector of atom $i$, and $\Phi_{i j k}^{\alpha \beta \gamma}$ are the third-order force constants. The mass-disorder (md) scattering rate is given by [67]

$$
\frac{1}{\tau_{\lambda}^{\mathrm{md}}}=\frac{\pi}{2} \sum_{\lambda^{\prime}} \omega_{\lambda} \omega_{\lambda^{\prime}} \sum_{i} g_{i}\left|\boldsymbol{\epsilon}_{\lambda}^{i \dagger} \cdot \boldsymbol{\epsilon}_{\lambda^{\prime}}^{i}\right|^{2} \delta\left(\omega_{\lambda}-\omega_{\lambda^{\prime}}\right),
$$

where $\epsilon_{\lambda^{\prime}}^{i}$ is the polarization vector and $g_{i}$ is the mass-variance parameter. For the unmixed compounds, $\tau_{\lambda}^{\text {md }}$ only contains the effect of natural isotopes, while for mixed compounds it also includes the distribution of isovalent mixing. These effects are both captured in $g_{i}$, but it should be noted that the natural isotope contribution is considerably smaller than the contribution of the mixing distribution [61,67].

We also investigate the effect of grain-boundary $(\mathrm{gb})$ scattering. The scattering rate of grain boundaries is given by [70]

$$
\frac{1}{\tau_{\lambda}^{\mathrm{gb}}}=\frac{v_{\lambda}}{d}
$$

where $v_{\lambda}$ is the phonon velocity and $d$ is the characteristic domain (grain) size. We denote the intrinsic LTC arising from the combined effect of three-phonon scattering and isotope scattering as $\kappa_{\ell}^{\text {int }}$, with the additional effect of md-scattering as $\kappa_{\ell}^{\mathrm{md}}$, and with gb-scattering added on top of that as $\kappa_{\ell}^{\mathrm{md}, \mathrm{gb}}$.

\subsection{Alloying Scheme}

The $122 \mathrm{HHs}$ in this study are based on the 74 stable compounds found by Feng et al. [71], as well as the additional 48 ones studied in Ref. [72]. Sublattice disorder is introduced by partially substituting with the element of the same group in the next period except for period 6 elements, which are substituted with period 5 elements. One exception, Te, is substituted with Se to omit the radioactive Po. For sublattice solid solutions, consisting solely of compounds predicted to be stable by Feng et al. [71] which are listed in the supplementary material (SM), we linearly interpolate the lattice parameter and the force constants of the parent compound with those of the fully substituted compound. Throughout the study, we use a $10 \%$ mixing concentration. For instance, for $\mathrm{Zr}_{0.9} \mathrm{Hf}_{0.1} \mathrm{NiSn}$, the $\mathrm{X}$-site effective mass is equal to $90 \% \mathrm{Zr}$ plus $10 \% \mathrm{Hf}$ isotopic mass. Likewise, the lattice parameter and the force constants consist of $90 \%$ of those calculated for ZrNiSn and 10\% of those for HfNiSn. For the remaining compounds, we use force constants and lattice parameter from the parent compounds to limit computational costs.

\subsection{Computational Details}

Density functional theory (DFT) calculations are done using the VASP [73-75] software package using the generalized gradient approximation (GGA) functional PBEsol [76,77]. The plane-wave cutoff is set to $600 \mathrm{eV}$. The electronic self-consistency cutoff is set at $10^{-6} \mathrm{eV}$. Atoms are relaxed until all forces fall below $1 \mathrm{meV} / \AA$. Second- and third-order force 
constants are extracted from fifty supercell configurations with $3 \times 3 \times 3$ repetitions of the primitive unit cell. For each configuration, atoms are displaced from equilibrium according to a canonical ensemble set to $T=300 \mathrm{~K}$. Realistic atomic displacements are calculated by assuming that the zero-point energy of the phonons corresponds to the Debye temperature [78]. The Debye temperature is obtained from the elastic tensor [79], which is calculated with DFT using the frozen phonon approach. In the supercell calculations, we use a $3 \times 3 \times 3 k$-point sampling of the Brillouin zone. The elastic tensor calculations are done with the primitive unit cell and an $11 \times 11 \times 11 k$-point sampling.

When extracting the force constants, the cutoff radii of the third-order and secondorder interactions are $\sim 2 \%$ and $\sim 12 \%$ larger than half the width of the supercell, respectively. These choices ensure that the force-constant matrices have equal sizes, allowing straightforward interpolation. The phonon-mode Brillouin zone integration in Equation (3) is sampled on a $35 \times 35 \times 35 q$-point mesh.

\section{Results and Analysis}

\subsection{Lattice thermal conductivity with Mass-Disorder and Grain-Boundary Scattering}

Figure $1 \mathrm{a}-\mathrm{c}$ shows the LTC with intrinsic scattering, $\kappa_{\ell}^{\text {int }}$, and with the additional md-scattering included, $\kappa_{\ell}^{\mathrm{md}}$, for the $122 \mathrm{HH}$ compounds. Figure $1 \mathrm{~d}$ plots $\kappa_{\ell}^{\mathrm{int}}$ versus $\kappa_{\ell}^{\mathrm{md}}$, where the color of the triangles indicates substitution site, displaying the reduction in LTC for each of the substitutions. On average, $X$-site substitution reduces the LTC by $43 \%$, somewhat less than the $\sim 49 \%$ reduction at $Y$ - and Z-sites. There is a substantial variation in $\kappa_{\ell}^{\mathrm{md}}$ when substituting the different sites, as shown by the large vertical spread of the triangles and the distributed bins of the histogram. To exemplify, partial X-site substitution of VIrGe (Figure 1c) reduces the LTC by $14 \%$, while the reduction with Z-site substitution in LiBSi (Figure 1a) is $80 \%$. The site-dependence can also differ greatly from one compound to the next. In the case of $\mathrm{ZrRhSb}$ and $\mathrm{ZrPdPb}$ (Figure 1c), the reduction is quite similar for the three different substitution sites, while for VFeSb (Figure 1c), X- and $Z$-site partial substitution leads to a reduction of $21 \%$ and $70 \%$, respectively. In total, we find 91 compounds with more than a $25 \%$ difference between the largest and smallest $\kappa_{\ell}^{\mathrm{md}}$ obtained for the different substitution sites.

Based on this data, it is interesting to formulate some basic guidelines on which substitution site to select to most efficiently reduce the LTC. One simple rule, which we previously noted as a trend in Ref. [80], is to substitute on the site hosting the heaviest atom. This rule gives the correct result for 102 out of the 122 compounds. Among the 20 compounds not following this rule, there are only 4 cases in which the $\kappa_{\ell}^{\text {md }}$ obtained with heaviest-site substitution and optimal-site substitution differ by more than $15 \%$. These 4 counterexamples can be explained by the fact that the mass-variance parameter $g_{i}$ of the optimal-site substitutions for these compounds is more than $50 \%$ larger than that of the heaviest-site substitution.

Another possible rule for attaining the largest LTC reduction could be to use the substitution with the highest $g_{i}$. In general, however, this turns out to be less efficient than substituting on sites with heavy atoms; often a high $g_{i}$ coincides with lighter atoms, which are associated with high-frequency phonon contributions to heat transport [61]. This is exemplified by $\mathrm{NbCoPb}$, which is optimally substituted on the Z-site. Given the relatively similar mass of $\mathrm{Pb}$ and $\mathrm{Sn}, \mathrm{Z}$-site substitution results in merely $g_{Z}=0.02$; the lighter Co partially substituted by $\mathrm{Rh}$ yields, on the other hand, $g_{Y}=0.04$, while $\mathrm{Nb}$ substituted by Ta corresponds to $g_{X}=0.07$.

Figure 1e shows the effect of introducing gb-scattering on the LTC, including optimalsite substitutions. The relative drop in LTC is larger for compounds with high $\kappa_{\ell}^{\text {int }}$. For the 20 compounds with lowest $\kappa_{\ell}^{\text {int }}$, the reduction in LTC using $100 \mathrm{~nm}$ grains and substitution on the optimal site is on average $69 \%$, while for the 20 compounds with the highest $\kappa_{\ell}^{\text {int }}$, the average reduction is $85 \%$. When optimal-site substitution has been included, reducing the grain size from $100 \mathrm{~nm}$ to $50 \mathrm{~nm}$ only gives a modest reduction in LTC; the average drop compared to the intrinsic LTC changes from $79 \%$ to $83 \%$. 

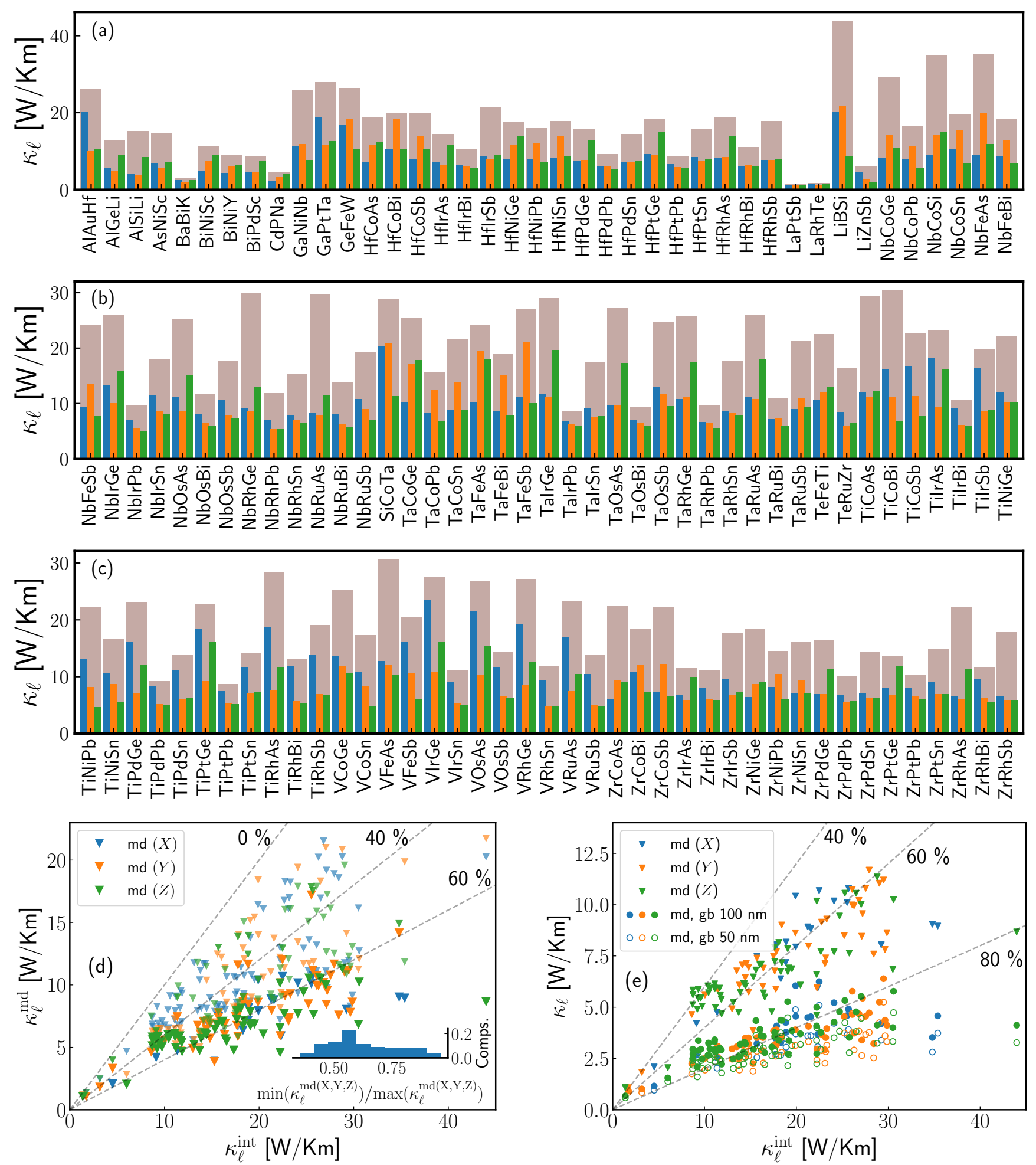

Figure 1. The (a-c) panels compare the LTC involving only intrinsic scattering $\kappa_{\ell}^{\text {int }}$ (grey bars) with that obtained from the addition of md-scattering on sites $X, Y$, and $Z$ (blue, orange, green), i.e., $\kappa_{\ell}^{\mathrm{md}(X)}$ at $300 \mathrm{~K}$. Dots mark the optimal substitution site. Panel (d) plots $\kappa_{\ell}^{\text {int }}$ against $\kappa_{\ell}^{\mathrm{md}}$. The blue, orange, and green triangles indicate substitution on the $X_{-}, Y_{-}$, and Z-sites, respectively, with the one most effectively reducing the LTC highlighted. The inset histogram shows the distribution of the most effective substitution divided by the least effective one, $\min \left(\kappa_{\ell}^{\operatorname{md}(X, Y, Z)}\right) / \max \left(\kappa_{\ell}^{\operatorname{md}(X, Y, Z)}\right)$. In panel (e), only results for the most effective substitution site are shown; in addition filled (empty) discs indicate $\kappa_{\ell}^{\mathrm{md}, \mathrm{gb}}$ with gb size of $100 \mathrm{~nm}(50 \mathrm{~nm})$. 
Grain boundaries most efficiently scatter less energetic acoustic phonons [62,81], while mass disorder scatters more energetic phonons $[61,82,83]$. It is therefore interesting to assess the reduction achieved with gb-scattering without the inclusion of md-scattering. We find that introducing gb-scattering with grain sizes of 100 and $50 \mathrm{~nm}$ reduces LTC on average by $42 \%$ and $54 \%$, respectively, see details in the SM. This reduction is hence more modest than that of $10 \%$ substitution on the optimal site.

\subsection{Compounds with Low Lattice Thermal Conductivity}

Certain compounds reach very low LTC with the introduction of md- and gb-scattering. Some of these are simply compounds that already have a low intrinsic LTC, such as $\mathrm{LaPtSb}, \mathrm{LaRhTe}, \mathrm{BaBiK}$, and CdPNa. For these compounds, it is interesting to gauge whether additional scattering mechanisms provide a substantial reduction in the LTC. Others are compounds which exhibits a significant drop in LTC with the inclusion of additional scattering mechanisms. It is useful to identify the key characteristics of these compounds, for the sake of developing effective thermoelectric optimisation and material identification strategies.

In Figure 2, we compare the intrinsic LTC, $\kappa_{\ell}^{\text {int }}$, with the LTC where md-scattering has been included, $\kappa_{\ell}^{\mathrm{md}}$, for the compounds with lowest $\kappa_{\ell}^{\mathrm{md}}$ at temperatures $300 \mathrm{~K}, 500 \mathrm{~K}$, and $700 \mathrm{~K}$. As a reference, $\mathrm{NbCoSn}, \mathrm{ZrNiSn}$, and the average LTCs for all HHs are also included. For partial substitution on the optimal site (reducing LTC the most), we also display the effect of gb-scattering with a grain size of $100 \mathrm{~nm}$.

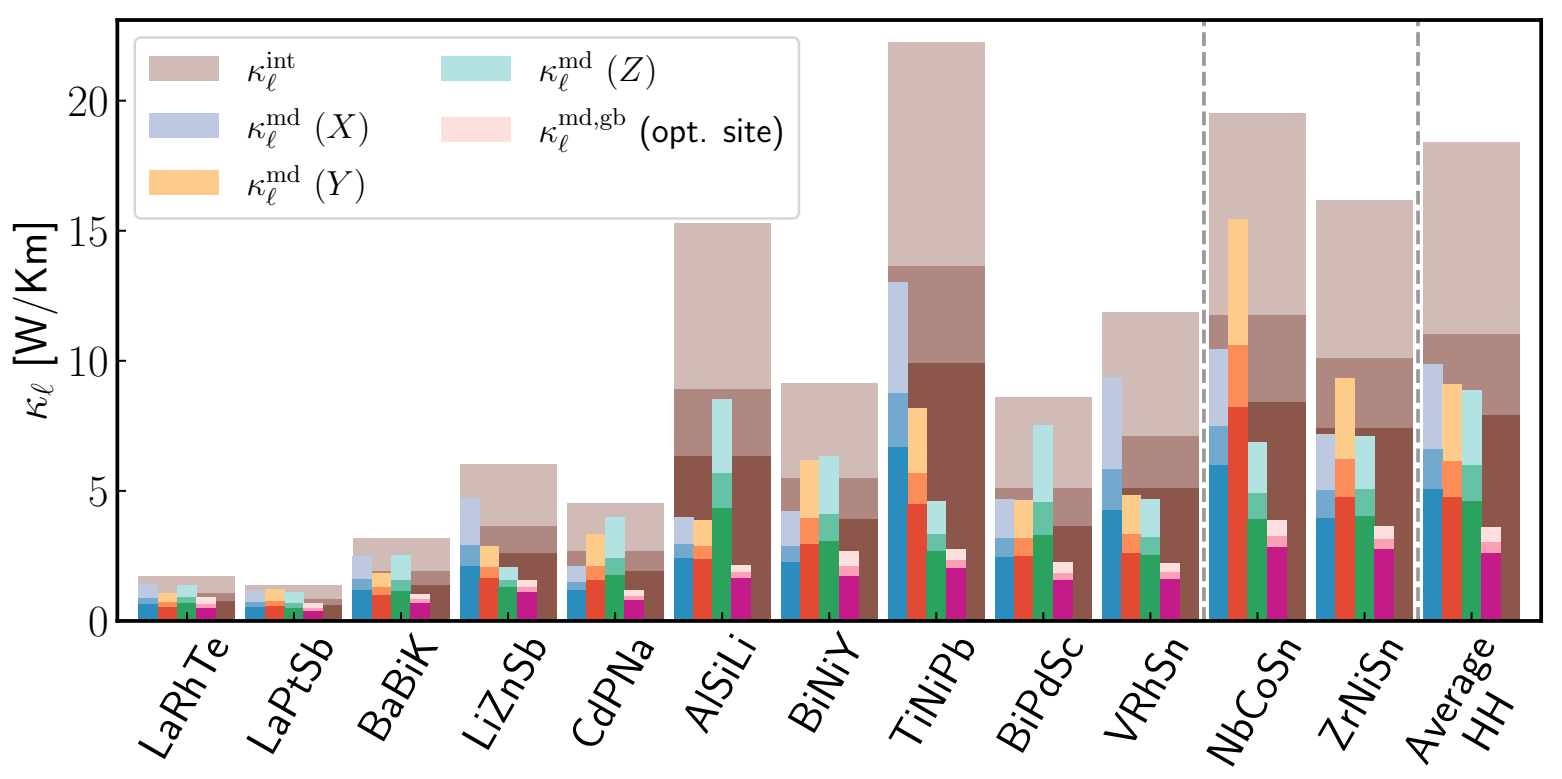

Figure 2. $\kappa_{\ell}^{\mathrm{int}}$ and $\kappa_{\ell}^{\mathrm{md}}$ for the $10 \mathrm{HH}$ compounds with the lowest $\kappa_{\ell}^{\mathrm{md}}$ at $300 \mathrm{~K}$, compared with $\mathrm{NbCoSn}, \mathrm{ZrNiSn}$, and the average of all $\mathrm{HH}$ compounds as reference. Brown bars indicate the intrinsic LTC, while blue, orange, and green bars indicate the LTC with substitutions on $X_{-}, Y_{-}$, and Z-sites. Sub-bars of increasing color intensity indicate the LTC at $300 \mathrm{~K}, 500 \mathrm{~K}$, and $700 \mathrm{~K}$. Purple bars indicate $\kappa_{\ell}^{\mathrm{md}, \mathrm{gb}}$, where substitution is done on the site reducing LTC the most, including gb-scattering using a domain size of $100 \mathrm{~nm}$.

For the compounds with the largest potential drop in LTC, there is a strong substitution site sensitivity. In general, we find that these compounds are characterized by a large mass difference between the heaviest and lightest atoms in the parent compound, with substitution on the heaviest atom being preferable. This can be exemplified by TiNiPb and AlSiLi, which exhibit the largest potential drop in LTC with the introduction of mdscattering; $\mathrm{Pb}$ is much heavier than $\mathrm{Ti}$ and $\mathrm{Ni}$, and $\mathrm{Al}$ and $\mathrm{Si}$ are much heavier than $\mathrm{Li}$. TiNiPb has a relatively high $\kappa_{\ell}^{\text {int }}=22.2 \mathrm{~W} / \mathrm{Km}$ at $300 \mathrm{~K}$, but $Z$-site substitution brings it 
down to $\kappa_{\ell}^{\mathrm{md}}=4.6 \mathrm{~W} / \mathrm{Km}$, whereas $X$-site substitution results in $13.0 \mathrm{~W} / \mathrm{Km}$ and $Y$-site results in $8.2 \mathrm{~W} / \mathrm{Km}$. AlSiLi has the largest reduction with $Y$-site substitution, resulting in $\kappa_{\ell}^{\mathrm{md}}=3.9 \mathrm{~W} / \mathrm{Km}$, quite similar to that of $X$-site substitution, which results in $4.0 \mathrm{~W} / \mathrm{Km}$, while Z-site substitution results in $8.5 \mathrm{~W} / \mathrm{Km}$.

For the compounds with lowest $\kappa_{\ell}^{\text {int }}$, i.e., LaPtSb, LaRhTe, BaBiK, and CdPNa, substitution on the optimal site reduces, on average, the LTC by $38 \%$, while the average reduction for all the HHs is $58 \%$. Thus, while the reduction achieved by introducing additional scattering mechanisms is lower for these compounds, it is still substantial. It is also interesting to compare $\mathrm{LiZnSb}$ and $\mathrm{CdPNa}$. Both these compounds have a quite similar mass-variance parameter $g_{i}$ at the optimal substitution site: $g_{i}=0.05$ for substitution on the $X$-site in CdPNa and $g_{i}=0.04$ for Z-site substitution in LiZnSb. Nonetheless, the effect of substitution is far stronger for LiZnSb. Similar to AlSiLi, LiZnSb is characterized by one of the atoms being much lighter than the other two.

When gb-scattering is introduced in addition to md-scattering, LiZnSb, AlSiLi, BiPdSc, and VRhSn achieve $\kappa_{\ell}^{\mathrm{md} \text {,gb }} \leq 2.2 \mathrm{~W} / \mathrm{Km}$, comparable to the values of the compounds with the lowest intrinsic LTC without such scattering mechanisms. In comparison, $\mathrm{NbCoSn}$ and $\mathrm{ZrNiSn}$ have $\kappa_{\ell}^{\mathrm{md} \text {,gb }}$ of $3.9 \mathrm{~W} / \mathrm{Km}$ and $3.6 \mathrm{~W} / \mathrm{Km}$, and the average for the HHs of this study is $\kappa_{\ell}^{\mathrm{md}, \mathrm{gb}}=3.6 \mathrm{~W} / \mathrm{Km}$ when using optimal-site substitutions.

Figure 3 shows the change in $\kappa_{\ell}^{\text {md,gb }}$ with temperature. While the relative ordering of low to high $\kappa_{\ell}^{\mathrm{md}, \mathrm{gb}}$ compounds tends to be retained with temperature, there is some difference in the relative reduction with increasing temperatures for different compounds. Interestingly, the compounds having a low intrinsic LTC, LaPtSb, LaRhTe, BaBiK, and $\mathrm{CdPNa}$, have a relatively large reduction in $\kappa_{\ell}^{\mathrm{md}, \mathrm{gb}}$ with temperature, on average $44 \%$ when going from 300 to $800 \mathrm{~K}$. The average reduction for all HHs is only $32 \%$ over the same temperature span. The compounds that attain a low $\kappa_{\ell}^{\mathrm{md}, \mathrm{gb}}$, LiZnSb, AlSiLi, BiPdSc, VRhSn, $\mathrm{BiNiY}$, and $\mathrm{TiNiPb}$, have an average reduction of $33 \%$, i.e., similar to that of the average for the HHs. Among these, the largest reduction is found for BiNiY, for which the LTC decreases by $40 \%$ when going from 300 to $800 \mathrm{~K}$. In general $\kappa_{\ell}^{\mathrm{md} \text {,gb }}$ shows a more modest reduction with temperature than $\kappa_{\ell}^{\text {int }}$, which on average is reduced by $62 \%$. The slight differences between the temperature dependence from one compound to the next, on the other hand, almost vanish without the presence of additional scattering mechanisms.

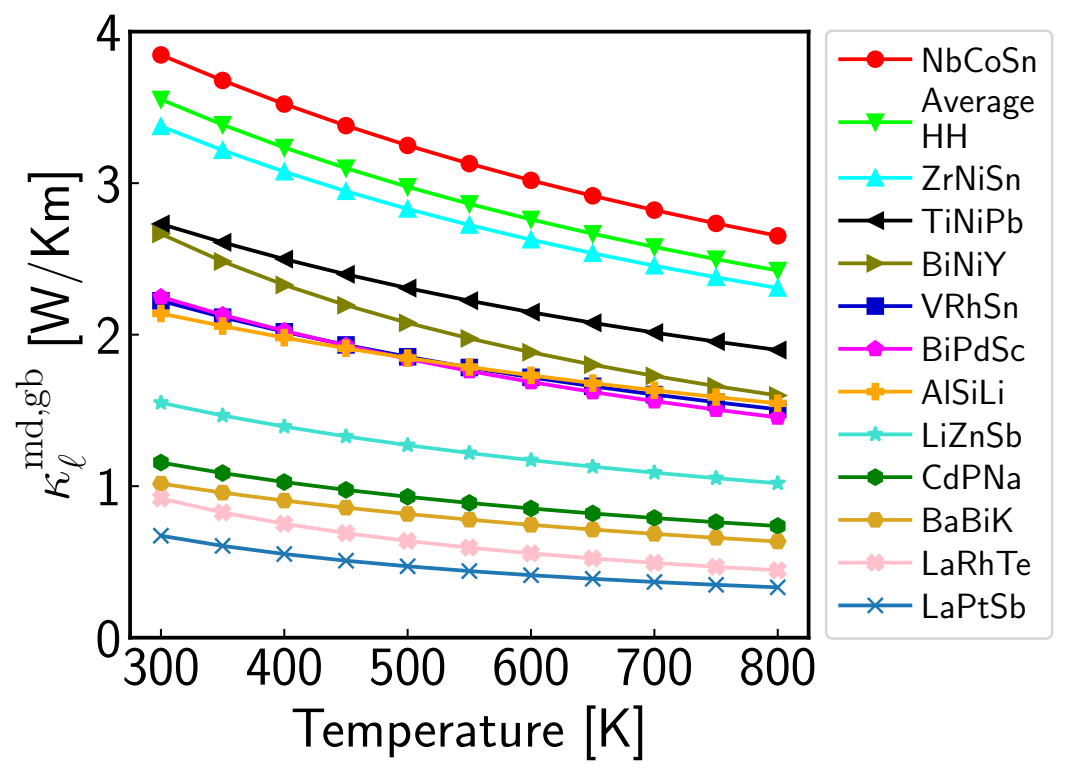

Figure 3. $\kappa_{\ell}^{\mathrm{md}, \mathrm{gb}}$ as a function of temperature, with substitution on the optimal site and a domain size of $100 \mathrm{~nm}$. The temperature is indicated on the horizontal axis, and $\kappa_{\ell}^{\mathrm{md}, \mathrm{gb}}$ on the vertical axis. 


\section{Discussion}

While our paper provides valuable guidance on the optimization of sublattice solid solutions for reducing the LTC, there are certain limitations of our analysis to bear in mind. First, the study is based on the virtual crystal approximation, in which the sublattice disorder is not accounted for explicitly. For a selection of the compounds, we simply use the average "virtual" mass on a site, while for others we also interpolate the force constants and lattice parameters, as discussed in Section 2.2. These limitations imply uncertainty in the calculated numbers.

Second, we neglect strain-induced scattering when the mass-disorder effect is considered. In a recent paper, Arrigoni et al. [84] showed that force-constant disorder can in some systems significantly reduce the predicted LTC. For $\operatorname{In}_{0.5} \mathrm{Ga}_{0.5} \mathrm{As}$ the reduction was $\sim 40 \%$, while for $\mathrm{Si}_{0.3} \mathrm{Ge}_{0.7}$ it was less than $10 \%$. The difference between the LTC obtained with interpolation of the mass, lattice parameter, and force constants compared with interpolation of only the mass provides some indication of the magnitude of the strain-induced scattering. This is because a large shift would imply that force constants of the two compounds are quite different, indicating large strain-induced scattering matrix elements. As detailed in the $\mathrm{SM}$, we find that interpolating the lattice parameter and force constants changes the LTC on average by $6.6 \%$ for substitution on the $X$-site, $7.3 \%$ for the $Y$-site, and $7.2 \%$ for the Z-site. Thus, while this change is modest, it is not insubstantial; for some compounds, e.g., for $Y$-site substituted $\mathrm{NbCoSn}$ it is quite large, having a relative deviation of $23 \%$. Nonetheless, md-scattering provides a rough lower bound of the scattering introduced through substitution. One could argue that a large mass-variance tends to correlate with variance in the effective size of the atoms and as such strain induced-scattering. Thus, one could hope that much of the strain-induced scattering would be described indirectly by the mass-variance. However, strain affects more atom sites than the mass-disorder, which is entirely local. The relative difference in the effect of substitution on different sites would therefore be somewhat affected by strain. The significance of this is difficult to evaluate, but we assume that the key trends identified here remain valid.

Third, we use a substitution concentration of $10 \%$ throughout this work. Increasing the concentration could further reduce LTC, but in terms of synthesis, higher concentrations can also lead to phase separation $[66,85]$. We do note, however, that phase separation could in some cases be beneficial for thermoelectric performance [86]. Sublattice miscibility is not considered in this paper, but several $\mathrm{HH}$ alloys demonstrate substitution concentrations higher than $10 \%[59,60,62,66,87,88]$. However, some cases, such as $10 \%$ substitution of $\mathrm{Li}$ with $\mathrm{Na}$ in $X$-site substituted LiZnSb could be unfeasible.

Given the many approximations, we perform a rough comparison with experiment to obtain an indication of the accuracy of the predictions. In doing so, we first consider that the computed intrinsic LTC is often much larger than the experimental LTC, especially for unmixed compounds $[61,62,72]$. This overestimation can, at least partially, be explained by the many impurities and defects present in real-world samples [35,89-91]. To correct for this, we first compute an effective contribution to the thermal conductivity arising from mdscattering, based on Matthiessen's rule: $1 / \kappa_{\ell}^{\prime}=1 / \kappa_{\ell}^{\text {md }}-1 / \kappa_{\ell}^{\text {int }}$. This expression can then be compared with a corresponding experimental quantity, $1 / \kappa_{\ell, \exp }^{\prime}=1 / \kappa_{\ell, \exp }^{\text {mixed }}-1 / \kappa_{\ell, \exp }^{\text {parent }}$. An underlying assumption in this comparison is that the phonon scattering due to impurities and other defects not related to mixing is quite similar in the mixed $\left(\kappa_{\ell, \text { exp }}^{\text {mixed }}\right)$ and pure compound $\left(\kappa_{\ell, \exp }^{\text {parent }}\right)$. Under this assumption, it is meaningful to compare $\kappa_{\ell, \exp }^{\prime}$ with the theoretical counterpart $\kappa_{\ell}^{\prime}$, allowing for a rough comparison of the computed effect of mdscattering with the mixing induced experimental effect. Comparing $X$-site substitution in ZrNiSn at $500 \mathrm{~K}$, we obtain in this work $\kappa_{\ell}^{\prime}=9.9 \mathrm{~W} / \mathrm{Km}$, which is in reasonable agreement with the experimental value of $\kappa_{\ell, \exp }^{\prime}=7.0 \mathrm{~W} / \mathrm{Km}$ for $\mathrm{Zr}_{0.9} \mathrm{Hf}_{0.1} \mathrm{NiSn}$ [59]. Moreover, for $X$-site substitution in VFeSb at $300 \mathrm{~K}$, we find $\kappa_{\ell}^{\prime}=21.8 \mathrm{~W} / \mathrm{Km}$, which can be compared with $\kappa_{\ell, \exp }^{\prime}=18.7 \mathrm{~W} / \mathrm{Km}$ [92] for $\mathrm{V}_{0.9} \mathrm{Nb}_{0.1} \mathrm{FeSb}$. These differences are sizeable but still 
point to the general validity of using md-scattering as a rough indicator of the effect of mixing on the LTC.

We also present results both with and without gb-scattering. The gb-scattering model is highly idealized, assuming perfect scattering of long-wavelength acoustic phonons. Real-world materials have grains of different sizes and orientations which scatter phonons differently. Domain sizes of 50 and $100 \mathrm{~nm}$ are assumed here, while depending on the preparation method, experimentally average grain sizes have been reported in the 60 to $400 \mathrm{~nm}$ range $[62,93,94]$. Nonetheless, gb-scattering provides some indication of how grains and other macroscopic defects filter out the contributions of mostly long-wavelength phonons to the LTC.

On a final note, one must keep in mind that substitutions and grain boundaries also impact electron transport. The additional electron scattering introduced causes a reduction in the electron mobility and in turn the conductivity $\sigma$ [60]. However, the subsequent reduction in $Z T$ would be lessened by the simultaneous reduction in the electronic thermal conductivity $\kappa_{e}$. LaPtSb, LaRhTe, BaBiK, and CdPNa have $\kappa_{\ell}^{\mathrm{md}, \mathrm{gb}} \leq 1.2 \mathrm{~W} / \mathrm{Km}$, comparable to well-known low LTC thermoelectric materials such as PbTe $[95,96]$ and SnSe $[10,97]$. This value is similar to or even lower than $\kappa_{e}$ in several doped HHs [80], e.g., $\kappa_{e}=1.4 \mathrm{~W} / \mathrm{Km}$ for $\mathrm{NbCo}_{0.94} \mathrm{Ni}_{0.06} \mathrm{Sn}$ [98] and $\kappa_{e}=1.5 \mathrm{~W} / \mathrm{Km}$ for $\mathrm{ZrPtSn}_{0.92} \mathrm{Sb}_{0.0 .08}$ [99]. Thus, with the identification of low LTC comparable to $\kappa_{e}$, emphasis can shift to design strategies that reduce $\kappa_{e}$.

\section{Conclusions}

This study has investigated the effect of isovalent sublattice-substitutions and grain boundaries on the lattice thermal conductivity (LTC) of 122 half-Heusler compounds. Calculations were done using density functional theory and the temperature-dependent effective potential method. We have shown that several compounds with relatively high LTC could be promising thermoelectric materials because of a substantial reduction in LTC with the introduction of additional phonon scattering mechanisms. In general, the largest reduction in LTC was achieved when substituting on the site hosting the heaviest atom rather than substituting on the site hosting the atom having the largest mass-variance parameter. We identified four compounds with quite large intrinsic LTC; LiZnSb, AlSiLi, BiPdSc, and VRhSn, for which the LTC fell below $2.2 \mathrm{~W} / \mathrm{Km}$ at $300 \mathrm{~K}$ with the introduction of mass-disorder and grain-boundary scattering. For the four compounds with the lowest intrinsic LTC, we found that both mass-disorder and grain-boundary scattering can further reduce LTC, although the relative reduction is smaller, resulting in an LTC below $1.2 \mathrm{~W} / \mathrm{Km}$.

Our study highlights that the presence of elements with large masses can be beneficial for thermoelectric materials. Such elements both reduce phonon velocities and allow for large mass-disorder scattering. However, compounds with heavy atoms are typically associated with expensive and rare elements like $\mathrm{La}, \mathrm{Pt}$, and $\mathrm{Pd}$, or toxic elements such as $\mathrm{Pb}$ and $\mathrm{Te}$. This makes them less desirable for new large-scale thermoelectric applications. However, the low LTC of Ga-substituted AlSiLi and Bi-substituted LiZnSb illustrates that it can be sufficient that one or two of the atoms are substantially heavier than the others once mass-disorder and grain-boundary scattering effects are taken into account. It is interesting to note that the reason for the large drop in LTC seen for such compounds could be connected to the nature of phonon modes. The substantial mass difference in zinc-blende compounds has been linked to large gaps between acoustic and optical phonons and small three-phonon scattering phase-space volume [100]. Such compounds would therefore typically have intrinsic LTC on the higher end, and thus potentially a large reduction in LTC with the introduction of additional scattering mechanisms. The identification of compounds with a large drop in LTC with the introduction of additional scattering mechanisms highlights the importance of going beyond three-phonon scattering when screening materials for thermoelectric applications. The prospect of finding efficient thermoelectric materials that are also cheap, easy to manufacture, and non-toxic remains bright. 
Supplementary Materials: The following are available online at https:/ / www.mdpi.com/article / 10.3390/electronicmat3010001/s1.

Author Contributions: Conceptualization, K.B.; software, R.T.; formal analysis, R.T. and K.B.; investigation, R.T.; data curation, R.T.; writing-original draft preparation, R.T.; writing-review and editing, O.M.L. and K.B.; visualization, R.T. and K.B.; supervision, O.M.L. and K.B.; project administration, K.B.; funding acquisition, O.M.L. and K.B. All authors have read and agreed to the published version of the manuscript.

Funding: The computations in this work were funded by The Norwegian e-infrastructure for research and education, Sigma2, through grants No. NN9711K and NN2615K. This work is part of the Allotherm project (Project No. 314778) funded by the Research Council of Norway.

Institutional Review Board Statement: Not applicable.

Informed Consent Statement: Not applicable.

Data Availability Statement: Data is contained within the article or Supplementary Material.

Conflicts of Interest: The authors declare no conflict of interest.

Abbreviations
The following abbreviations are used in this manusc
HH half-Heusler
DFT density functional theory
LTC lattice thermal conductivity
TDEP temperature-dependent effective potential
md mass-disorder
gb
SM $\quad$ grain-boundary

\section{References}

1. Ando Junior, O.; Maran, A.; Henao, N. A review of the development and applications of thermoelectric microgenerators for energy harvesting. Renew. Sust. Energ. Rev. 2018, 91, 376-393. [CrossRef]

2. Champier, D. Thermoelectric generators: A review of applications. Energy Convers. Manag. 2017, 140, 167-181. [CrossRef]

3. Snyder, G.; Toberer, E. Complex thermoelectric materials. Nat. Mater. 2008, 7, 105-114. [CrossRef] [PubMed]

4. Zhu, T.; Liu, Y.; Fu, C.; Heremans, J.P.; Snyder, J.G.; Zhao, X. Compromise and Synergy in High-Efficiency Thermoelectric Materials. Adv. Mater. 2017, 29, 1605884. [CrossRef] [PubMed]

5. Twaha, S.; Zhu, J.; Yan, Y.; Li, B. A comprehensive review of thermoelectric technology: Materials, applications, modelling and performance improvement. Renew. Sust. Energ. Rev. 2016, 65, 698-726. [CrossRef]

6. Wongprakarn, S.; Pinitsoontorn, S.; Tanusilp, S.A.; Kurosaki, K. Enhancing thermoelectric properties of p-type SiGe alloy through optimization of carrier concentration and processing parameters. Mater. Sci. Semicond. Process 2018, 88, 239-249. [CrossRef]

7. Yang, L.; Chen, Z.G.; Dargusch, M.; Zou, J. High performance thermoelectric materials: Progress and their applications. Adv. Energy Mater. 2017, 8, 1701797. [CrossRef]

8. Gutierrez Moreno, J.; Cao, J.; Fronzi, M.; Assadi, M.H.N. A review of recent progress in thermoelectric materials through computational methods. Mater. Renew. 2020, 9, 16. [CrossRef]

9. Gangjian, T.; Shi, F.; Hao, S.; Zhao, L.; Chi, H.; Zhang, X.; Uher, C.; Wolverton, C.; Dravid, V.; Kanatzidis, M. Non-equilibrium processing leads to record high thermoelectric figure of merit in PbTe-SrTe. Nat. Commun. 2016, 7, 12167. [CrossRef]

10. Zhou, C.; Lee, Y.K.; Yu, Y.; Byun, S.; Luo, Z.Z.; Lee, H.; Ge, B.; Lee, Y.L.; Chen, X.; Lee, J.Y.; et al. Polycrystalline SnSe with a thermoelectric figure of merit greater than the single crystal. Nat. Mater. 2021, 20. [CrossRef]

11. Wheatley, R.; Roble, M.; Gence, L.; Acuña, C.; Rojas-Aedo, R.; Hidalgo-Rojas, D.; Guzman-De La Cerda, D.; Vojkovic, S.; Seifert, B.; Wallentowitz, S.; et al. Structural, optoelectronic and photo-thermoelectric properties of crystalline alloy $\mathrm{CuAl}_{x} \mathrm{Fe}_{1-x} \mathrm{O}_{2}$ delafossite oxide materials. J. Alloys Compd. 2021, 857, 157613. [CrossRef]

12. Rajagopal, A.; Stoddard, R.J.; Hillhouse, H.W.; Jen, A.K.Y. On understanding bandgap bowing and optoelectronic quality in $\mathrm{Pb}-\mathrm{Sn}$ alloy hybrid perovskites. J. Mater. Chem. A 2019, 7, 16285-16293. [CrossRef]

13. Nikam, R.; Kwak, M.; Lee, J.; Rajput, K.; Banerjee, W.; Hwang, H. Near ideal synaptic functionalities in Li ion synaptic transistor using $\mathrm{Li}_{3} \mathrm{PO}_{x} \mathrm{Se}_{x}$ electrolyte with high ionic conductivity. Sci. Rep. 2019, 9, 18883. [CrossRef] [PubMed]

14. Zevgolis, A.; Wood, B.C.; Mehmedović, Z.; Hall, A.T.; Alves, T.C.; Adelstein, N. Alloying effects on superionic conductivity in lithium indium halides for all-solid-state batteries. APL Mater. 2018, 6, 047903. [CrossRef] 
15. Haseman, M.S.; Karim, M.R.; Ramdin, D.; Noesges, B.A.; Feinberg, E.; Jayatunga, B.H.D.; Lambrecht, W.R.L.; Zhu, M.; Hwang, J.; Kash, K.; et al. Deep level defects and cation sublattice disorder in ZnGeN2. J. Appl. Phys. 2020, 127, 135703. [CrossRef]

16. Novikov, V.V.; Matovnikov, A.V.; Mitroshenkov, N.V.; Shevelkov, A.V.; Bud'ko, S.L. Crystal lattice disorder and characteristic features of the low-temperature thermal properties of higher borides. Dalton Trans. 2020, 49, 2138-2144. [CrossRef] [PubMed]

17. Marlton, F.P.; Zhang, Z.; Zhang, Y.; Proffen, T.E.; Ling, C.D.; Kennedy, B.J. Lattice Disorder and Oxygen Migration Pathways in Pyrochlore and Defect-Fluorite Oxides. Chem. Mater. 2021, 33, 1407-1415. [CrossRef]

18. Zhang, X.; Wang, H.; Hickel, T.; Rogal, J.; Li, Y.; Neugebauer, J. Mechanism of collective interstitial ordering in Fe-C alloys. Nat. Mater. 2020, 19, 849-854. [CrossRef]

19. Zhang, Y.Y.; Mishra, R.; Pennycook, T.J.; Borisevich, A.Y.; Pennycook, S.J.; Pantelides, S.T. Oxygen disorder, a way to accommodate large epitaxial strains in oxides. Adv. Mater. Interfaces 2015, 3, 1500344. [CrossRef]

20. Wang, X.; Ling, Y.; Lian, X.K.; Xin, Y.; Dhungana, K.B.; Perez-Orive, F.; Knox, J.M.; Chen, Z.; Zhou, Y.; Beery, D.; et al. Suppressed phase separation of mixed-halide perovskites confined in endotaxial matrices. Nat. Commun. 2019, 10, 695. [CrossRef]

21. Švecová, I.; Tillová, E.; Kuchariková, L.; Knap, V. Possibilities of predicting undesirable iron intermetallic phases in secondary Al-alloys. Transp. Res. Procedia 2021, 55, 797-804. [CrossRef]

22. Sopiha, K.V.; Larsen, J.K.; Donzel-Gargand, O.; Khavari, F.; Keller, J.; Edoff, M.; Platzer-Björkman, C.; Persson, C.; Scragg, J.J.S. Thermodynamic stability, phase separation and $\mathrm{Ag}$ grading in $(\mathrm{Ag}, \mathrm{Cu})(\mathrm{In}, \mathrm{Ga}) \mathrm{Se}_{2}$ solar absorbers. J. Mater. Chem. A 2020, 8, 8740-8751. [CrossRef]

23. Berche, A.; Tédenac, J.C.; Jund, P. Phase separation in the half-Heusler thermoelectric materials (Hf,Ti,Zr)NiSn. Scr. Mater. 2017, 139, 122-125. [CrossRef]

24. Synoradzki, K.; Ciesielski, K.; Kepiński, L.; Kaczorowski, D. Effect of secondary LuNiSn phase on thermoelectric properties of half-Heusler alloy LuNiSb. Mater. Today Proc. 2019, 8, 567-572. [CrossRef]

25. Gürth, M.; Rogl, G.; Romaka, V.; Grytsiv, A.; Bauer, E.; Rogl, P. Thermoelectric high ZT half-Heusler alloys $\mathrm{Ti}_{1-x-y} \mathrm{Zr}_{x} \mathrm{Hf}_{y} \mathrm{NiSn}$ $(0 \leq x \leq 1 ; 0 \leq y \leq 1)$. Acta Mater. 2016, 104, 210-222. [CrossRef]

26. Dalpian, G.M.; Zhao, X.G.; Kazmerski, L.; Zunger, A. Formation and Composition-Dependent Properties of Alloys of Cubic Halide Perovskites. Chem. Mater. 2019, 31, 2497-2506. [CrossRef]

27. Chen, L.; Tan, Y.Y.; Chen, Z.X.; Wang, T.; Hu, S.; Nan, Z.A.; Xie, L.Q.; Hui, Y.; Huang, J.X.; Zhan, C.; et al. Toward Long-Term Stability: Single-Crystal Alloys of Cesium-Containing Mixed Cation and Mixed Halide Perovskite. J. Am. Chem. Soc. 2019, 141, 1665-1671. [CrossRef] [PubMed]

28. Sato, M.; Chai, Y.W.; Kimura, Y. Effect of Half-Heusler Interfacial Structure on Thermal Transport Properties of (Ti, Zr)NiSn Alloys. ACS Appl. Mater. Interfaces 2021, 13, 25503-25512. [CrossRef] [PubMed]

29. Bae, K.W.; Hwang, J.Y.; Kim, S.i.; Jeong, H.M.; Kim, S.; Lim, J.H.; Kim, H.S.; Lee, K.H. Thermoelectric Transport Properties of n-Type Sb-doped (Hf,Zr,Ti)NiSn Half-Heusler Alloys Prepared by Temperature-Regulated Melt Spinning and Spark Plasma Sintering. Appl. Sci. 2020, 10, 4963. [CrossRef]

30. El-Khouly, A.; Novitskii, A.; Adam, A.; Sedegov, A.; Kalugina, A.; Pankratova, D.; Karpenkov, D.; Khovaylo, V. Transport and thermoelectric properties of Hf-doped FeVSb half-Heusler alloys. J. Alloys Compd. 2020, 820, 153413. [CrossRef]

31. Bos, J.W.; Downie, R. Half-Heusler thermoelectrics: A complex class of materials. J. Condens. Matter Phys. 2014, $26,433201$. [CrossRef] [PubMed]

32. Luo, T.; Serrano-Sánchez, F.; Bishara, H.; Zhang, S.; Bueno Villoro, R.; Kuo, J.J.; Felser, C.; Scheu, C.; Snyder, G.J.; Best, J.P.; et al. Dopant-segregation to grain boundaries controls electrical conductivity of n-type $\mathrm{NbCo}(\mathrm{Pt}) \mathrm{Sn}$ half-Heusler alloy mediating thermoelectric performance. Acta Mater. 2021, 217, 117147. [CrossRef]

33. Fu, C.; Bai, S.; Liu, Y.; Tang, Y.; Chen, L.; Zhao, X.; Zhu, T. Realizing high figure of merit in heavy-band $p$-type half-Heusler thermoelectric materials. Nat. Commun. 2015, 6, 8144. [CrossRef] [PubMed]

34. He, R.; Kraemer, D.; Mao, J.; Zeng, L.; Jie, Q.; Lan, Y.; Li, C.; Shuai, J.; Kim, H.S.; Liu, Y.; et al. Achieving high power factor and output power density in $p$-type half-Heuslers $\mathrm{Nb}_{1-x} \mathrm{Ti}_{x} \mathrm{FeSb}$. PNAS 2016, 113, 13576-13581. [CrossRef]

35. Katre, A.; Carrete, J.; Mingo, N. Unraveling the dominant phonon scattering mechanism in thermoelectric compound ZrNiSn. J. Mater. Chem. A 2016, 4, 15940-15944. [CrossRef]

36. Naydenov, G.; Hasnip, P.; Lazarov, V.; Probert, M. Huge power factor in p-type half-Heusler alloys NbFeSb and TaFeSb. J. Phys. Mater. 2019, 2, 035002. [CrossRef]

37. Khandy, S. Inspecting the electronic structure and thermoelectric power factor of novel p-type half-Heuslers. Sci. Rep. 2021, 11, 1. [CrossRef]

38. Carrete Montaña, J.; Mingo, N.; Wang, S.; Curtarolo, S. Nanograined half-Heusler semiconductors as Advanced Thermoelectrics: An Ab Initio High-Throughput Statistical Study. Adv. Funct. Mater. 2014, 24, 7427-7432. [CrossRef]

39. El-Khouly, A.; Adam, A.; Ibrahim, E.; Nafady, A.; Karpenkov, D.; Novitskii, A.; Voronin, A.; Khovaylo, V.; Elsehly, E. Mechanical and thermoelectric properties of FeVSb-based half-Heusler alloys. J. Alloys Compd. 2021, 886, 161308. [CrossRef]

40. Ning, S.; Huang, S.; Zhang, Z.; Zhang, R.; Qi, N.; Chen, Z. High thermoelectric performance of topological half-Heusler compound LaPtBi achieved by hydrostatic pressure. Phys. Chem. Chem. Phys. 2020, 22, 14621-14629. [CrossRef]

41. Shiomi, J.; Esfarjani, K.; Chen, G. Thermal conductivity of half-Heusler compounds from first-principles calculations. Phys. Rev. B 2011, 84, 104302. [CrossRef] 
42. Hoat, D. Electronic structure and thermoelectric properties of Ta-based half-Heusler compounds with 18 valence electrons. Comput. Mater. Sci. 2019, 159, 470-477. [CrossRef]

43. Fava, M.; Dongre, B.; Carrete, J.; van Roekeghem, A.; Madsen, G.K.H.; Mingo, N. Effects of doping substitutions on the thermal conductivity of half-Heusler compounds. Phys. Rev. B 2021, 103, 174112. [CrossRef]

44. Sun, H.L.; Yang, C.L.; Wang, M.S.; Ma, X.G. Remarkably High Thermoelectric Efficiencies of the Half-Heusler Compounds BXGa (X = Be, Mg, and Ca). ACS Appl. Mater. Interfaces 2020, 12, 5838-5846. [CrossRef]

45. Gaultois, M.W.; Sparks, T.D. How much improvement in thermoelectric performance can come from reducing thermal conductivity? Appl. Phys. Lett. 2014, 104, 113906. [CrossRef]

46. Wolf, M.; Hinterding, R.; Feldhoff, A. High power factor vs. high zT-A review of thermoelectric materials for high-temperature application. Entropy 2019, 21, 1058. [CrossRef]

47. Mao, J.; Wang, Y.; Kim, H.S.; Liu, Z.; Saparamadu, U.; Tian, F.; Dahal, K.; Sun, J.; Chen, S.; Liu, W.; et al. High thermoelectric power factor in $\mathrm{Cu}-\mathrm{Ni}$ alloy originate from potential barrier scattering of twin boundaries. Nano Energy 2015, 17, 279-289. [CrossRef]

48. Skoug, E.; Zhou, C.; Pei, Y.; Morelli, D.T. High thermoelectric power factor in alloys based on CoSi. Appl. Phys. Lett. 2009, 94, 022115. [CrossRef]

49. Heinrich, C.; Day, T.; Zeier, W.; Snyder, G.; Tremel, W. Effect of isovalent substitution on the thermoelectric properties of the $\mathrm{Cu}_{2} \mathrm{ZnGeSe}_{4-x} \mathrm{~S}_{x}$ series of solid solutions. J. Am. Chem. Soc. 2013, 136, 442-448. [CrossRef] [PubMed]

50. Sun, H.; Lu, X.; Morelli, D.T. Isovalent substitutes play in different ways: Effects of isovalent substitution on the thermoelectric properties of $\mathrm{CoSi}_{0.98} \mathrm{~B}_{0.02}$. J. Appl. Phys. 2016, 120, 035107. [CrossRef]

51. Chmielowski, S.; Bhattacharya, S.; Jacob, S.; Péré, D.; Jacob, A.; Moriya, K.; Delatouche, B.; Roussel, P.; Madsen, G.; Dennler, G. Strong reduction of thermal conductivity and enhanced thermoelectric properties in $\mathrm{CoSbS}_{1-x} \mathrm{Se}_{x}$ paracostibite. Sci. Rep. 2017, 7, 46630. [CrossRef]

52. Shuai, J.; Wang, Y.; Liu, Z.; Kim, H.S.; Mao, J.; Sui, J.; Ren, Z. Enhancement of thermoelectric performance of phase pure Zintl compounds $\mathrm{Ca}_{1-x} \mathrm{Yb}_{x} \mathrm{Zn}_{2} \mathrm{Sb}_{2}, \mathrm{Ca}_{1-x} \mathrm{Eu}_{x} \mathrm{Zn}_{2} \mathrm{Sb}_{2}$, and $\mathrm{Eu}_{1-x} \mathrm{Yb}_{x} \mathrm{Zn}_{2} \mathrm{Sb}_{2}$ by mechanical alloying and hot pressing. Nano Energy 2016, 25, 136-144. [CrossRef]

53. Zevalkink, A.; Pomrehn, G.S.; Johnson, S.; Swallow, J.; Gibbs, Z.M.; Snyder, G.J. Influence of the triel elements (M = Al, Ga, In) on the transport properties of $\mathrm{Ca}_{5} \mathrm{M}_{2} \mathrm{Sb}_{6}$ Zintl compounds. Chem. Mater. 2012, 24, 2091-2098. [CrossRef]

54. Guo, M.; Guo, F.; Zhu, J.; Yin, L.; Zhang, Q.; Cai, W.; Sui, J. Achieving high thermoelectric performance in rare-earth element-free $\mathrm{CaMg}_{2} \mathrm{Bi}_{2}$ with high carrier mobility and ultralow lattice thermal conductivity. Research 2020, 2020, 1-10. [CrossRef]

55. Zhou, J.; Huang, L.; Wang, Z.; Liu, Z.; Cai, W.; Sui, J. Effect of Cd isoelectronic substitution on thermoelectric properties of $\mathrm{Zn}_{0.995} \mathrm{Na}_{0.005}$ Sb. J. Mater. 2016, 2, 324-330. [CrossRef]

56. Zheng, W.; Lu, Y.; Li, Y.; Wang, J.; Hou, Z.; Shao, X. Structural and thermoelectric properties of Zr-doped TiPdSn half-Heusler compound by first-principles calculations. Chem. Phys. Lett. 2020, 741, 137055. [CrossRef]

57. Zhang, J.; Huang, L.; Zhu, C.; Zhou, C.; Jabar, B.; Li, J.; Zhu, X.; Wang, L.; Song, C.; Xin, S.; et al. Design of domain structure and realization of ultralow thermal conductivity for record-high thermoelectric performance in chalcopyrite. Adv. Mater. 2019, 31, 1905210. [CrossRef]

58. Yu, J.; Fu, C.; Liu, Y.; Xia, K.; Aydemir, U.; Chasapis, T.; Snyder, G.; Zhao, X.; Zhu, T. Unique role of refractory Ta alloying in enhancing the figure of merit of $\mathrm{NbFeSb}$ thermoelectric materials. Adv. Energy Mater. 2017, 8, 1701313. [CrossRef]

59. Chauhan, N.S.; Bathula, S.; Vishwakarma, A.; Bhardwaj, R.; Johari, K.K.; Gahtori, B.; Saravanan, M.; Dhar, A. Compositional tuning of ZrNiSn half-Heusler alloys: Thermoelectric characteristics and performance analysis. J. Phys. Chem. 2018, 123, 105-112. [CrossRef]

60. Mallick, M.; Rajput, K.; Vitta, S. Increasing figure-of-merit of ZrNiSn half-Heusler alloy by minimal substitution and thermal conductivity reduction. J. Mater. Sci. Mater. 2019, 30, 6139-6147. [CrossRef]

61. Eliassen, S.N.H.; Katre, A.; Madsen, G.K.H.; Persson, C.; Løvvik, O.M.; Berland, K. Lattice thermal conductivity of $\mathrm{Ti}_{x} \mathrm{Zr}_{y} \mathrm{Hf}_{1-x-y} \mathrm{NiSn}$ half-Heusler alloys calculated from first principles: Key role of nature of phonon modes. Phys. Rev. B 2017, 95, 045202. [CrossRef]

62. Schrade, M.; Berland, K.; Eliassen, S.; Guzik, M.; Echevarria-Bonet, C.; Sørby, M.; Jenus, P.; Hauback, B.; Tofan, R.; Gunnæs, A.; et al. The role of grain boundary scattering in reducing the thermal conductivity of polycrystalline $\mathrm{XNiSn}(\mathrm{X}=\mathrm{Hf}$, Zr, Ti) half-Heusler alloys. Sci. Rep. 2017, 7, 1. [CrossRef]

63. Yan, R.; Xie, W.; Balke, B.; Chen, G.; Weidenkaff, A. Realizing p-type NbCoSn half-Heusler compounds with enhanced thermoelectric performance via Sc substitution. Sci. Technol. Adv. Mater. 2020, 21, 122-130. [CrossRef] [PubMed]

64. Ono, Y.; Inayama, S.; Adachi, H.; Kajitani, T. Thermoelectric properties of NbCoSn-based half-Heusler alloys. In Proceedings of the 2006 25th International Conference on Thermoelectrics, Vienna, Austria, 6-10 August 2006; pp. 124-127. [CrossRef]

65. Kumar, A.; Chaturvedi, K.M.; Bano, S.; Govind, B.; Misra, D. Enhanced thermoelectric performance of p-type ZrCoSb $\mathrm{Zn}_{0.9} \mathrm{Sn}_{0.1}$ via Tellurium doping. Mater. Chem. Phys. 2021, 258, 123915. [CrossRef]

66. Yuan, B.; Wang, B.; Huang, L.; Lei, X.; Zhao, L.; Wang, C.; Zhang, Q. Effects of Sb Substitution by Sn on the Thermoelectric Properties of ZrCoSb. J. Electron. Mater. 2016, 46. [CrossRef]

67. Tamura, S.I. Isotope scattering of dispersive phonons in Ge. Phys. Rev. B 1983, 27, 858-866. [CrossRef] 
68. Hellman, O.; Abrikosov, I.A. Temperature-dependent effective third-order interatomic force constants from first principles. Phys. Rev. B 2013, 88, 144301. [CrossRef]

69. Hellman, O.; Abrikosov, I.A.; Simak, S.I. Lattice dynamics of anharmonic solids from first principles. Phys. Rev. B 2011, 84, 180301. [CrossRef]

70. Srivastava, G. The Physics of Phonons; Taylor \& Francis: Abingdon, UK, 1990.

71. Feng, Z.; Fu, Y.; Zhang, Y.; Singh, D.J. Characterization of rattling in relation to thermal conductivity: Ordered half-Heusler semiconductors. Phys. Rev. B 2020, 101, 064301. [CrossRef]

72. Tranås, R.; Løvvik, O.M.; Tomic, O.; Berland, K. Lattice thermal conductivity of half-Heuslers with density functional theory and machine learning: Enhancing predictivity by active sampling with principal component analysis. Comput. Mater. Sci. 2022, 202, 110938. [CrossRef]

73. Kresse, G.; Hafner, J. Ab initio molecular dynamics for liquid metals. Phys. Rev. B 1993, 47, 558-561. [CrossRef] [PubMed]

74. Kresse, G.; Furthmüller, J. Efficiency of ab-initio total energy calculations for metals and semiconductors using a plane-wave basis set. Comput. Mater. Sci. 1996, 6, 15-50. [CrossRef]

75. Kresse, G.; Furthmüller, J. Efficient iterative schemes for ab initio total-energy calculations using a plane-wave basis set. Phys. Rev. B 1996, 54, 11169-11186. [CrossRef] [PubMed]

76. Perdew, J.P.; Ruzsinszky, A.; Csonka, G.I.; Vydrov, O.A.; Scuseria, G.E.; Constantin, L.A.; Zhou, X.; Burke, K. Restoring the density-gradient expansion for exchange in solids and surfaces. Phys. Rev. Lett. 2008, 100, 136406. [CrossRef]

77. Csonka, G.I.; Perdew, J.P.; Ruzsinszky, A.; Philipsen, P.H.T.; Lebègue, S.; Paier, J.; Vydrov, O.A.; Ángyán, J.G. Assessing the performance of recent density functionals for bulk solids. Phys. Rev. B 2009, 79, 155107. [CrossRef]

78. Shulumba, N.; Hellman, O.; Minnich, A.J. Intrinsic localized mode and low thermal conductivity of PbSe. Phys. Rev. B 2017, 95, 014302. [CrossRef]

79. Anderson, O. A simplified method for calculating the debye temperature from elastic constants. J. Phys. Chem. Solids 1963, 24, 909-917. [CrossRef]

80. Berland, K.; Shulumba, N.; Hellman, O.; Persson, C.; Løvvik, O.M. Thermoelectric transport trends in group 4 half-Heusler alloys. J. Appl. Phys. 2019, 126, 145102. [CrossRef]

81. Goldsmid, H.; Penn, A. Boundary scattering of phonons in solid solutions. Phys. Lett. A 1968, 27, 523-524. [CrossRef]

82. Tian, Z.; Lee, S.; Chen, G. Heat Transfer in Thermoelectric Materials and Devices. J. Heat Transfer 2013, 135, 061605. [CrossRef]

83. Xu, Q.; Zhou, J.; Liu, T.H.; Chen, G. Effect of electron-phonon interaction on lattice thermal conductivity of SiGe alloys. Appl. Phys. Lett. 2019, 115, 023903. [CrossRef]

84. Arrigoni, M.; Carrete, J.; Mingo, N.; Madsen, G.K.H. First-principles quantitative prediction of the lattice thermal conductivity in random semiconductor alloys: The role of force-constant disorder. Phys. Rev. B 2018, 98, 115205. [CrossRef]

85. Geng, H.; Zhang, H. Effects of phase separation on the thermoelectric properties of (Ti, Zr, Hf)NiSn half-Heusler alloys. J. Appl. Phys. 2014, 116, 033708. [CrossRef]

86. Rausch, E.; Balke, B.; Stahlhofen, J.M.; Ouardi, S.; Burkhardt, U.; Felser, C. Fine tuning of thermoelectric performance in phase-separated half-Heusler compounds. J. Mater. Chem. C 2015, 3, 10409-10414. [CrossRef]

87. Serrano-Sánchez, F.; Luo, T.; Yu, J.; Xie, W.; Le, C.; Auffermann, G.; Weidenkaff, A.; Zhu, T.; Zhao, X.; Alonso, J.A.; et al. Thermoelectric properties of n-type half-Heusler NbCoSn with heavy-element Pt substitution. J. Mater. Chem. A 2020, 8 , 14822-14828. [CrossRef]

88. Chauhan, N.S.; Bathula, S.; Vishwakarma, A.; Bhardwaj, R.; Johari, K.K.; Gahtori, B.; Dhar, A. Enhanced thermoelectric performance in p-type $\mathrm{ZrCoSb}$ based half-Heusler alloys employing nanostructuring and compositional modulation. J. Mater. 2019, 5, 94-102. [CrossRef]

89. Hazama, H.; Matsubara, M.; Asahi, R.; Takeuchi, T. Improvement of thermoelectric properties for half-Heusler TiNiSn by interstitial Ni defects. J. Appl. Phys. 2011, 110, 063710. [CrossRef]

90. Xie, H.; Wang, H.; Fu, C.; Liu, Y.; Snyder, G.; Zhao, X.; Zhu, T. The intrinsic disorder related alloy scattering in ZrNiSn half-Heusler thermoelectric materials. Sci. Rep. 2014, 4, 6888. [CrossRef]

91. Ferluccio, D.A.; Kennedy, B.F.; Barczak, S.A.; Popuri, S.R.; Murray, C.; Pollet, M.; Bos, J.W.G. Thermal properties of TiNiSn and VFeSb half-Heusler thermoelectrics from synchrotron x-ray powder diffraction. J. Phys. Energy 2021, 3, 035001. [CrossRef]

92. Fu, C.; Xie, H.; Zhu, T.J.; Xie, J.; Zhao, X.B. Enhanced phonon scattering by mass and strain field fluctuations in $\mathrm{Nb}$ substituted FeVSb half-Heusler thermoelectric materials. J. Appl. Phys. 2012, 112, 124915. [CrossRef]

93. Cho, J.; Park, T.; Bae, K.; Kim, H.S.; Choi, S.M.; Kim, S.I.; Kim, S. Ti Addition Effect on the Grain Structure Evolution and Thermoelectric Transport Properties of $\mathrm{Hf}_{0.5} \mathrm{Zr}_{0.5} \mathrm{NiSn}_{0.98} \mathrm{Sb}_{0.02}$ Half-Heusler Alloy. Materials 2021, 14, 4029. [CrossRef] [PubMed]

94. Zhao, H.; Cao, B.; Li, S.; Liu, N.; Shen, J.; Li, S.; Jian, J.; Gu, L.; Pei, Y.; Snyder, G.; et al. Engineering the Thermoelectric Transport in Half-Heusler Materials through a Bottom-Up Nanostructure Synthesis. Adv. Energy Mater. 2017, 7, 1700446. [CrossRef]

95. Su, C.H. Experimental determination of lattice thermal conductivity and Lorenz number as functions of temperature for n-type PbTe. Mater. Today Phys. 2018, 5, 58-63. [CrossRef]

96. Lu, Y.; Sun, T.; Zhang, D.B. Lattice anharmonicity, phonon dispersion, and thermal conductivity of PbTe studied by the phonon quasiparticle approach. Phys. Rev. B 2018, 97, 174304. [CrossRef]

97. Xiao, Y.; Chang, C.; Pei, Y.; Wu, D.; Peng, K.; Zhou, X.; Gong, S.; He, J.; Zhang, Y.; Zeng, Z.; et al. Origin of low thermal conductivity in SnSe. Phys. Rev. B 2016, 94, 125203. [CrossRef] 
98. Yan, R.; Xie, R.; Xie, W.; Shen, C.; Li, W.; Balke, B.; Yoon, S.; Zhang, H.; Weidenkaff, A. Effects of Doping Ni on the Microstructures and Thermoelectric Properties of Co-Excessive NbCoSn Half-Heusler Compounds. ACS Appl. Mater. Interfaces 2021, 13, 34533-34542. [CrossRef]

99. Dai, C.K.; Song, Q.F.; Xie, L.; Liu, R.H.; Bai, S.; Chen, L.D. Improving thermoelectric properties of ZrPtSn-based half-Heusler compound by Sb doping. Rare Met. 2021, 40, 2838-2846. [CrossRef]

100. Lee, S.; Esfarjani, K.; Luo, T.; Zhou, J.; Tian, Z.; Chen, G. Resonant bonding leads to low lattice thermal conductivity. Nat. Commun. 2014, 5, 3525. [CrossRef] [PubMed] 